\title{
Jörg MEIER, Georg SCHUPPENER: Werbesprache in der Mitte Europas. Nationale Elemente \\ in der Produktwerbung im Vergleich. \\ (= Beiträge zur Fachdidaktik 2). \\ Wien: Praesens Verlag, 2018: $61 \mathrm{~S}$.
}

Heutzutage scheint die Werbung omnipräsent zu sein und man wagt es überhaupt nicht, sich das Leben ohne Werbung vorzustellen. Mehr noch, die Werbung ist zu einem festen Bestandteil unserer Existenz geworden, sie prägt unsere Entscheidungen und beeinflusst alltägliche Tätigkeiten, obwohl es uns oft nicht so sehr bewusst sein mag. Daher verwundert es auch nicht, dass Werbung als Textsorte auch im Unterricht gerne und oft didaktisiert wird. Einen Katalog der Anregungen für die Didaktisierung der Werbung bietet die Monographie von Jörg Meier und Georg Schuppener u.d.T.: Werbesprache in der Mitte Europas. Nationale Elemente in der Produktwerbung im Vergleich, die im Jahre 2018 im Praesens Verlag in der Reihe Beiträge zur Fachdidaktik erschienen ist.

Die Autoren der Monographie gehen davon aus, dass sich in der Werbung Schrift und Bild verbinden und sie sich daher besonders gut für didaktische Zwecke eignet, zumal Werbetexte zur Förderung der Lesekompetenz bei Schülern beitragen können. Aus diesem Grunde betrachten sie Werbetexte als eine Fundgrube für didaktische Anregungen diverser Art. Da Werbetexte auch im Leben der Lernenden vorkommen, ist auch der Zugang leichter, denn dadurch kann auch ihr Interesse daran entwickelt werden. Die Verfasser betrachten die Werbetexte als Mittel, die dazu verhelfen sollen, ,einen modernen und praxisnahen DeutschUnterricht zu realisieren“ (S. 8) und verfolgen zugleich die Absicht, mit ihrem methodisch ausgerichteten Werk eine Lücke in diesem Bereich zu schließen. Die Monographie besteht aus sieben Kapiteln, einem Resümee und dem Literaturverzeichnis. 
Im ersten Kapitel werden Grundlagen und Voraussetzungen der Werbesprachenforschung thematisiert. Die Verfasser weisen dabei auf die Allgegenwärtigkeit der Werbung hin, was sich auch darin niederschlägt, dass sie als „Abbild des gegenwärtigen Sprachgebrauchs“ (S. 9) betrachtet werden kann. Besprochen werden auch Eigenschaften und Funktionen dieser Textsorte, die sie als eine besondere Gattung des Alltags ausmachen.

Den Gegenstand des zweiten Teils bilden die Sprache und Geschichte der Werbung. Das Kapitel liefert in synthetischer Form einen Überblick über die Entwicklung der Werbung seit der 2. Hälfte des 19. Jahrhunderts. Besondere Aufmerksamkeit wird dabei dem Einfluss der sich ständig entwickelnden Massenmedien geschenkt, die auf jeder Etappe die Werbelandschaft wesentlich geprägt haben. Im Zusammenhang damit unterscheiden die Autoren einige Phasen in der Evolution dieser Gattung. Die erste Etappe wird in der Nachkriegszeit verortet, in der die Werbung eher plakativ war. Die steigende Konkurrenz beeinflusste jedoch die späteren Veränderungen im Bereich der Werbung und war die Ursache für die zweite Phase der Entwicklung: Man warb nicht nur für das Produkt, das im Zentrum stand, sondern hob auch dessen Qualitäten hervor. In den 60er Jahren des 20. Jahrhunderts erfolgte ein Durchbruch - es wurden Images und Leitbilder mit Menschen um das Produkt herum verkauft. In den 80er Jahren des 20. Jahrhunderts wurde Werbung zum Konsumprodukt und ihre Entwicklung gipfelte in den 90er Jahren im Cross-Media-Marketing. Dabei weisen die Verfasser darauf hin, dass „Werbeanzeigen ein Spiegel ihrer jeweiligen Gesellschaft sind“ (S. 17), daher können sie auch als ein Zeugnis der Kultur und Geschichte betrachtet werden. Im Anschluss an diesen historischen Überblick werden auch die Merkmale der Werbesprache thematisiert.

Das dritte Kapitel ist der Besprechung von Forschungsfragen gewidmet. Zuerst werden Disziplinen aufgelistet, mit denen die Forschung im Bereich der Werbung zusammenarbeitet. Im Fokus der Analyse stehen nationale Bezugnahmen in den Werbeprospekten, deren Bedeutung und Funktionen untersucht werden. Die Verfasser formulieren eine Reihe von Forschungsfragen (S. 20 f.), die im Fokus ihrer Analyse stehen, weisen jedoch zugleich darauf hin, dass diese nur eine Basis für weitere Fragestellungen darstellen.

Im vierten Kapitel werden das Material und die Methoden präsentiert, wobei auch unterschiedliche Zugänge zum Korpus aufgezeigt werden.

Den Kern der Monographie bildet das fünfte Kapitel, in dem exemplarische Analysen enthalten sind. Es wird gezeigt, wie das sprachliche und bildliche Material aus den Werbeprospekten didaktisch umgesetzt 
werden kann. Schritt für Schritt werden Vorschläge für den Einsatz ausgewählter Werbeprospekte und Werbetexte im Unterricht besprochen. Ergänzt wird dieses Kapitel mit einem Teil, in dem didaktische Hinweise für den Unterricht thematisiert werden. Die Verfasser entwerfen dabei einen Katalog von Fragen in Bezug auf die behandelte Textsorte, die bei der Gestaltung des Unterrichts hilfreich sein können. Die Fortsetzung findet man im siebten Kapitel, in dem der Einsatz von Werbeprospekten im DaF-Unterricht diskutiert wird. Auch hier findet der Leser eine breite Palette an Möglichkeiten der Didaktisierung von Werbetexten. Es werden zugleich auch methodische Fragen erörtert.

Die Monographie wird mit den Schlussfolgerungen abgerundet, in denen auf weitere Perspektiven im Bereich der Didaktisierung von Werbung eingegangen wird.

Die Monographie von Jörg Meier und Georg Schuppener stellt in synthetischer Form einen methodisch fundierten Überblick über mögliche didaktische Zugänge zur Werbung und deren Einsatz im Unterricht dar. Das Werk kann nicht nur für Lehrer und Lerner, sondern auch für diejenigen interessant sein, die in der Forschung im Bereich der Werbung tätig sind, denn es ist zugleich ein wissenschaftliches Kompendium für jeden. Besonders wertvoll sind die didaktischen Hinweise und praktischen Beispiele mit Vorschlägen zur Didaktisierung von Werbeprospekten. Dieses Werk wird sicherlich von allen Interessierten als anregend wahrgenommen und praktisch erprobt.

Joanna Szczęk

(D) https:orcid.org/0000-0001-8721-6661

Universität Wrocław 